a live baby was made. Caesarean section through a Pfannenstiel incision revealed a posterior type III placenta praevia but no sign of accidental haemorrhage. A healthy girl was delivered. During examination of the ovaries a clot of blood was removed from the left paracolic gutter and considerable bleeding was apparent from the splenic area. A large left paramedian incision was added and the haemorrhage controlled by packs. Rapid splenectomy was then performed with double
ligation of the splenic artery proximal to the aneurysmal dilatation. Recovery was uncomplicated and the patient and her daughter were discharged on the twelfth day.

Rupture of splenic artery aneurysm during pregnancy has been reviewed in detail. ${ }^{2}$ The average age of patients is 32 and $80 \%$ of ruptures occur in the last trimester or in labour. The maternal mortality is $69 \%$ and the fetal mortality $97 \%$. Survival appears to be related to early diagnosis and treatment. The present case is only the third ${ }^{2-4}$ in which both mother and child survived and the first such case in Britain. It seems likely that this case was an example of a "double rupture," with a limited initial rupture producing symptoms and a latent period ${ }^{5}$ before the subsequent severe haemorrhage. This success can be attributed to early intervention, albeit on mistaken diagnostic grounds, and to the rapid implementation of resuscitative measures.

The diagnosis of ruptured splenic artery aneurysm should be borne in mind in any case of obstetric shock and immediate laparotomy should be carried out if it is considered a possibility.

We would like to thank Mr. G. T. Hammond for allowing us to report this case. -We are, etc.

DECLAN GIBBENS DAVID HEATH

Royal Hampshire County Hospital,

Winchester

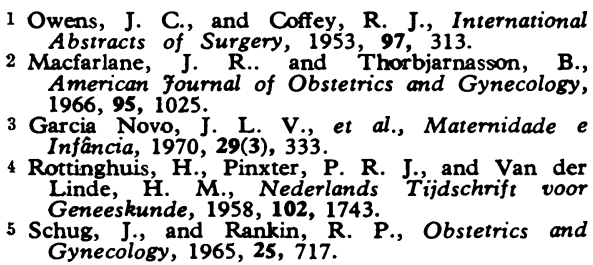

\section{Sleep Epilepsy}

SIR,-The findings of Drs. F. B. Gibberd and M. C. Bateson (25 May, p. 403) relative to the incidence, time of occurrence, and prognosis of sleep seizures are consistent with our experiences reported in $1954 .^{1}$ Our findings, however, are in contradistinction to their statement that "the age of onset in idiopathic daytime epilepsy is nearly always between 10 and 30 years." The most common age at onset in our group of more than 15,000 patients with idiopathic diurnal epilepsy was from four to seven years, with the highest incidence from five to six years. After the seventh year of age the onset of idiopathic epilepsy was distinctly less prevalent, though there was a moderate but significant increase in the 11 th and 12th years. Also, in contrast to the authors' statement that sleep seizures "tend to be symtomatic rather more often than daytime seizures," the incidence of symptomatic seizures in our series of patients with sleep epilepsy was considerably lower than that in our patients whose attacks oocurred only diurnally.
The prime purpose of this communication is to present our methods of treating sleep seizures, with particular emphasis on the use of dextroamphetamine sulphate, since Drs. Gibberd and Bateson did not discuss this subject specifically but recommended that "we should be cautious before using medication to suppress sleep seizures." We have found that sleep epilepsy is frequently resistant to the standard antiepileptic drugs such as phenobarbitone, phenytoin, and primidone; however, the following therapeutic regimens have proved effective in some patients.

For early nocturnal seizures (those which occur soon after falling asleep) the patient should initially be given the appropriate dosage of phenobarbitone three times a day. The first dose with lunch, the second with supper, and the third at bedtime. If the seizures should persist the second (supper) dose should be increased by increments of $32 \mathrm{mg}$, the first and third doses remaining the same, until a satisfactory control of seizures is attained or the limit of tolerance is reached. For late nocturnal or early morning seizures (those which occur shortly before the usual time of awakening or soon after awakening) the patient should be treated with phenobarbitone as above except that, if the seizures should persist or recur, it is the third (bedtime) dose that should be inoreased in increments of $32 \mathrm{mg}$. If phenobarbitone fails to control or appreciably reduce the frequency of the patient's seizures it should be gradually replaced by another major motor (grand mal) antiepileptic agent, preferably primidone; phenytoin is our drug of third choice for the treatment of sleep epilepsy.

We are definitely convinced, however, that the most efficacious drug for the control of sleep seizures is dextroamphetamine sulphate administered in a sustained release dosage form (Spansule) at bedtime. We have obtained very good and sometimes spectacular results with the following dosage schedules.

For older children and adolescents we institute dextroamphetamine therapy with a 5-mg Spansule taken at bedtime. If the seizures recur we raise the dosage to $10 \mathrm{mg}$ and, if necessary, to $15 \mathrm{mg}$. For adults therapy is inaugurated with a $15-\mathrm{mg}$ dextroamphetamine Spansule taken at bedtime. If this dosage proves ineffective we increase it to $25 \mathrm{mg}$.

We emphasize that our experience with the use of dextroamphetamine sulphate has demonstrated that side effects are minimal. Insomnia and anorexia, if present, generally disappear within a week or two after the initiation of therapy. In many of our patients dextroamphetamine was prescribed during childhood and continued for a period of years without weight loss, retardation of growth, or other significant side reactions, and on termination of therapy no evidence of serious withdrawal symptoms was noted.

For reasons that at present remain obscure, adults rather than infants or very young. children and patients with early rather than late nocturnal seizures respond better to dextroamphetamine therapy. In addition, we are unable to explain the mechanism of action of dextroamphetamine in controlling sleep seizures; it may be due to: (a) its anticonvulsant properties-4; (b) diminution of the depth of sleep, ${ }^{5}$ particularly stage IV; $(c)$ reduction of rapid eye movement sleep ${ }^{6} ;(d)$ increase in the proportion of time spent in stages II and II $\operatorname{sleep}^{7} ;(e)$ a combination of these effects; or $(f)$ other factors.-We are, etc.,

\section{SAMUEL Livingston} LYDIA L. PAULI

Samuel Livingston Epilepsy Diagnostic and Treatment Center,

1 Livingston, S., The Diagnosis and Treatment of Convulsive Disorders in Children, pp. 125-127. Springton, $S$ Kas, 1954 ,

fournal of Pediatrics, 1948, 32, 490

Goodman, L. S., and Gilman, A. The Pharmacological Basis of Therapeutics, p. 518 . New York, Macmillan. 1955 .

4 Preben, H., Ugeskrift for Laeger, 1958, 120, 1363 Livingston, S S Comprehensive Management of p. 99. Springfield, Thomas, 1972 .

Anders, T. F., and Weinstein, P., Pediatrics,

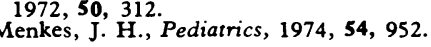

\section{Alcoholism}

SIR,-A sample census of alcoholics in the Brighton Health District has been made possible by the collaboration of my colleagues in general practice. This has resulted in an estimated total of 800 alcoholics known to their G.P.s in the Brighton and Hove area. The figures, which are completely anonymous, demonstrate that most are not skid-row characters but are poor devils who are trying to carry on a job or a home but are in the stranglehold of alcohol. They and their families do not need sermons, they need the help which does not at present exist.

Many potential patients in these circumstances do not consult their G.P.s My colleagues and I are therefore convinced that the figure of 800 for Brighton and Hove is a gross underestimate and that many more require support and reclamation.-I am, etc.,

W. S. PARKER District Community Physician,

Brighton Brighton Health District

\section{Practolol and the Eye}

SIR,-I read with great interest the letter of Mr. P. Wright (8 June, p. 560). One of my patients, a woman aged 62 years who has been suffering from angina for five years, has been taking practolol $400 \mathrm{mg}$ a day for 18 months. Recently she presented to me complaining of pain, photophobia, and diffioulty in opening the eyes. I referred her to an ophthalmologist who diagnosed keratoconjunctivitis sioca. She had dryness of the eye, and with rose Bengal there was no corneal staining but onjunctival staining with some filamentous ahanges in the conjunctiva and adhesions and obliteration of the lower fornices (essential atrophy of the conjunctiva). Practolol was stopped and she was put on methyl cellulose drops and Sofradex, with marked improvement. She had no skin eruptions.-I am, etc.,

Department of Cardiology,

SIDDIg A. ISMAIL

Khartoum, Sudan

Progestogen-only Oral Contraception and Ectopic Gestation

SIR,-Mr. J. Bonnar (16 February, p. 287) and Mr. D. F. Hawkins (2 March, p. 387) 